\title{
Metodología indirecta para la estimación de vida útil residual de transformadores de potencia a partir de la evaluación de los materiales dieléctricos Indirect methodology for life-span estimation of power transformers from the evaluation of its dielectric materials
}

Luis Diego Acuña-Barrantes ${ }^{1}$, Gustavo Adolfo Gómez-Ramírez²

Acuña-Barrantes, L; Gómez-Ramírez, G. Metodología indirecta para la estimación de vida útil residual de transformadores de potencia a partir de la evaluación de los materiales dieléctricos. Tecnología en Marcha. Vol. 33-3. Julio-Setiembre 2020. Pág 45-56.

doi) https://doi.org/10.18845/tm.v33i3.4485

Fecha de recepción: 29 de julio de 2019 Fecha de aprobación: 3 de noviembre de 2019 


\title{
Palabras clave
}

Aceite dieléctrico; papel dieléctrico; degradación; Furaldehídos; grado de polimerización.

\section{Resumen}

Una adecuada y confiable operación de los transformadores de potencia en las redes eléctricas constituye un factor determinante en la continuidad de servicio, lo cual justifica su atención en la evolución de su integridad, definida sobre todo por su sistema dieléctrico compuesto principalmente por papel - aceite. La investigación se centró en el análisis de pruebas químicas aplicadas a un grupo de transformadores de potencia de 10 MVA con distintos periodos de operación y su correlación con el envejecimiento real estimado por el Grado de Polimerización (GP) y el 2-furaldehído (2-FAL), además de pruebas de esfuerzo mecánico al papel dieléctrico. Los esfuerzos medidos en el papel dieléctrico presentaron una reducción de 74.49 \% respecto al valor mínimo establecido por la IEC (80 MPa) y un GP aproximado de 88.4. Las condiciones operativas de cada transformador junto con el porcentaje de humedad del papel dieléctrico, los gases totales y de Oxígeno medidos en ppm, permitieron estimar el 2-FAL de forma indirecta. Los resultados aproximados del Grado de Polimerización fueron suficientes para su evaluación como condición "levemente envejecida". De modo que, la aproximación de la vida útil residual máxima resultó ser de 27 años. Dicha estimación es congruente con los bajos niveles de gases combustibles medidos (menores a 700 ppm) y de Oxígeno.

\section{Keywords}

Dielectric oil; dielectric paper; degradation; Furaldehyde; degree of polymerization.

\begin{abstract}
An adequate and reliable operation of the power transformers in the electrical networks constitutes a determining factor in the continuity of service, which justifies its attention in the evolution of its integrity, defined by its dielectric system mainly conform by oil and paper. The research focused on the analysis of chemical tests applied to a group of 10 MVA power transformers with different periods of operation and their correlation with the actual aging estimated by the Degree of Polymerization (DP) and 2-furaldehyde (2-FAL), in addition to mechanical stress tests applied to the dielectric paper. This stress, measured in the dielectric paper, presented a reduction of $74.49 \%$ with respect to the minimum value established by the IEC ( $80 \mathrm{MPa})$ and a DP of 88.4. The operating conditions of each transformer along with the percentage of humidity of the dielectric paper, the total and Oxygen gases measured in ppm, allowed the estimation of 2-FAL indirectly. The approximate results of the Degree of Polymerization were enough for its evaluation as a "slightly aged" condition. So, the approximation of the maximum life-span was 27 years. This estimate is consistent with the low levels of measured combustible gases (less than 700 ppm) and Oxygen.
\end{abstract}

\section{Introducción}

El sistema dieléctrico en un transformador de potencia es el más sensible al deterioro y degradación, por lo cual es importante monitorear su evolución bajo las condiciones operativas [1]. El proceso de envejecimiento del papel dieléctrico es esencialmente una despolimerización o ruptura de cadenas celulósicas [2], causadas por efectos de humedad, temperatura y oxígeno presente en su ambiente, en este caso el aceite dieléctrico. Uno de sus mecanismos es la hidrólisis, donde el rompimiento de las cadenas de celulosa por da 
lugar a 3 moléculas adicionales de agua, además de $\mathrm{CO}$ y $\mathrm{CO}_{2}$ [2]. Además de disminuir sus propiedades mecánicas, la despolimerización se considera un proceso autocatalítico debido a la sinergia entre el agua y compuestos ácidos que dan como producto de reacción la liberación de moléculas de agua por cada unidad monosacárida de la cadena principal de Hemicelulosa [3]. Respecto a la oxidación, este proceso tiene lugar a bajas temperaturas (entre $30-120^{\circ} \mathrm{C}$ ) donde el oxígeno disuelto en el papel, activa las cadenas radicales junto con catalizadores como trazas metálicas o compuestos oxidables. Estos últimos son altamente reactivos en presencia de oxígeno. Su terminación es causada por una recombinación de especies radicales en cetonas y alcoholes [1]. Conforme se aumenta la temperatura, la pirólisis incrementa la cinética de reacción a temperaturas de envejecimiento más altas, lo que ocasiona un incremento gradual en su viscosidad como resultado de reacciones de policondensación. Los precipitados de este tipo de reacción son los lodos, los cuales son insolubles en el aceite [1]. La degradación del aceite dieléctrico, por otro lado, se da principalmente por oxidación. Al reaccionar en presencia de este elemento, se producen hidrocarburos de naturaleza oxidativa al contener este elemento [4], los cuales debido al contacto con el papel dieléctrico provocaran efectos negativos similares a los lodos. Estos precipitados se depositan en las bobinas, canales de refrigeración o en porosidades del papel dieléctrico y afectan la capacidad de transferencia de calor. Igualmente, comprometen la rigidez dieléctrica de los aislamientos al formar especies cargadas que, gracias a su alta polaridad, tienden a alinearse bajo campos eléctricos externos y favorecen el paso de cargas eléctricas (fugas o arcos internos). La combinación de factores que atenten directamente la integridad dieléctrica son los candidatos por excelencia para la detonación de fallas catastófricas en transformadores de potencia. Debido a su impacto tanto económico, ambiental o de servicio dentro de una red eléctrica, el uso de técnicas preventivopredictivas en mantenimiento han permitido controlar las variables que afectan directamente el equipo ante la detección temprana las fallas incipientes [5]. Los métodos indirectos implementados al papel-aceite dieléctrico son los más utilizados como fin de estimación de su valor de vida. Numerosos estudios han identificado que el deterioro del transformador de potencia se atribuye también a un deterioro de las propiedades mecánicas, en este caso del papel [6]. Esto argumenta la necesidad de conocer la calidad e integridad del aislante sólido por medio de su valor de Grado de Polimerización (GP) [3]. El valor promedio del número de anillos de Glucosa (moléculas de $\mathrm{C}_{6} \mathrm{H}_{10} \mathrm{O}_{5}$ ) representa el GP, que durante el envejecimiento tiende a disminuir debido al rompimiento de cadenas [3] [7]. Para el papel nuevo se consideran entre los 1300 a 1100 GP donde por lo general, tiende a disminuir a 950 una vez preparado el transformador para operar por primera vez (debido al proceso de vacío) [9]. Valores entre los 400 hasta 251 GP son considerados como el inicio de un envejecimiento crítico debido a la directa proporción existente a este punto entre las propiedades mecánicas y el Grado de Polimerización [9] [10]. La importancia de estas mediciones es su correlación con los productos de descomposición característicos de compuestos celulósicos, como los contenidos de Furanos o Furfural. El 2-furfural (2-FAL) ha mostrado una dependencia directa con las incisiones moleculares del papel y por medio de modelos matemáticos, es posible estimar valores de GP. Parte de las primeras investigaciones como [11], confirmaron la dependencia de la vida residual con la integridad del papel. Con el transcurso de más estudios en este campo, se evidenció que con la determinación del Grado de Polimerización (GP) fue posible modelar la evolución de su integridad, donde trabajos como [12] y [6] permitieron definir un límite admisible donde sus propiedades mecánicas no disminuyen drásticamente. Sin embargo, la presencia de factores externos comenzó a tener efecto en el deterioro, por ejemplo, en [13] se determinó que las aproximaciones cinéticas de tasas degradativas requerían considerar la presencia de oxígeno y humedad. De modo que, los avances en el campo como los de [11] [9], identificaron esta variación con la humedad, temperatura y contaminación de su ambiente. Esta correlación fundamentó distintas metodologías indirectas que permitían caracterizar los materiales dieléctricos y su proceso de degradación, algunas como el contenido de Furaldehídos (2-FAL) 
en el aceite y su relación con el GP como en [8], la relación de diferentes gases combustibles disueltos en el aceite con las posibles fallas eléctricas internas para el caso de [14] y otras consideraciones como las postuladas en [15]. Las intensivas investigaciones a partir de estos aspectos llevaron al estudio de la cinética de reacción y ser relacionarla con modelos matemáticos de estimación de vida residual. El modelo más utilizado es el de Arrhenius, que en [11] utilizan para la estimación de vida útil residual gracias la proporción inversa que existe con la tasa de reacción, temperatura y energía de activación del proceso de degradación. Por último, varios trabajos han tomado como base los modelos matemáticos anteriores pero ajustados según la presencia de humedad y Oxígeno, por medio de valores exponenciales en la ecuación [10], comprobados y postulados como factores de aceleración por [13]. Actualmente existen múltiples metodologías, aproximaciones y estimaciones, sean complementarias o específicas en el cálculo de vida útil residual, las cuales han sido postuladas, pero no evaluadas en aplicaciones reales. La investigación propuesta permite reunir modelos matemáticos para la estimación de vida útil residual con la inclusión de aspectos operativos y del sistema aceitepapel dieléctrico como indicadores indirectos. Además de predecir la condición remanente del transformador, en términos prácticos resulta ser una metodología versátil y económica al incorporar información de gases disueltos en el aceite, los cuales comúnmente se realizan, y no evaluaciones adicionales para la determinación de los niveles de Furfural. Igualmente, se plantearon pruebas mecánicas de tensión aplicadas al papel dieléctrico con el fin de demostrar el efecto degenerativo operativo y sus consecuencias.

\section{Metodología}

\section{Esfuerzo de Tensión mecánica}

Los ensayos mecánicos de tensión se aplicaron según la norma ASTM D828-16 a dos tipos de muestras de papel dieléctrico tipo Kraft; la primera como referencia (nueva) y la segunda de un transformador de potencia con 16 años de operación continua. Las pruebas se realizaron en el equipo MTS Bionix Servohydraulic del laboratorio de Mecánica de Materiales del Instituto Tecnológico de Costa Rica, con una celda de carga de $2.5 \mathrm{kN}$ y precarga de $5 \mathrm{~N}$, hasta alcanzar su esfuerzo último.

\section{Estimación del Grado de Polimerización a partir del ensayo de tensión mecánica}

La aproximación del grado de polimerización se efectuó por medio del reordenamiento de la ecuación propuesta por [6]:

$$
G P=\frac{3 * G P_{0}}{5} *\left(\frac{\sigma}{\sigma_{0}}-0.1\right) \quad(\text { ecuación } 1)
$$

donde $\sigma_{0}$ y $\sigma$ son los valores de esfuerzo de tensión inicial (80 MPa según [5]) y medido para el valor de GP respectivamente, $G P_{0}$ es el grado de polimerización inicial despúes del vacío interno en un transformador, con un valor de 950.

\section{Estimación de vida útil residual de transformadores de potencia}

El banco de resultados evaluados proviene de pruebas aplicadas a un grupo de transformadores de potencia monofásicos y trifásicos, todos a diferentes periodos operativos y con potencias nominales superiores a 10 MVA. Tanto las pruebas de gases disueltos como las de contenidos de furanos (2-FAL) disueltos fueron efectuadas según las normas ASTM D3612-02 (2017) y D5837-15 respectivamente. 
Aproximación indirecta del contenido de 2-FAL

La medición indirecta se realizó a partir la ecuación propuesta por [16]:

$$
\begin{aligned}
2 F A L[\mathrm{ppm}]= & -0.935+0.03018 * T+0.03399 * A+0.3883 * M+ \\
& 0.000048 * G_{O 2}-0.000023 * G_{\text {Total }}-0.000007 * C
\end{aligned} \text { (ecuación 2) }
$$

donde $T$ es la temperatura medida en el aceite dieléctrico en ${ }^{\circ} \mathrm{C}$, $A$ son los años de servicio del transformador, $M$ es el contenido de humedad del papel dieléctrico según las curvas de equilibrio propuestas por [17]. Los términos $G_{\mathrm{O} 2}$ y $G_{\text {Total }}$ son los contenidos de oxígeno y gases disueltos totales (ppm) respectivamente y $C$ es la capacidad del transformador en kVA.

\section{Estimación del Grado de Polimerización}

La evaluación del grado de polimerización del papel dieléctrico se basó en la ecuación propuesta por [18], en función del contenido de Furfural disuelto en el aceite dieléctrico:

$$
G P_{0}=\frac{8.88 * G P}{8.88+2 F A L} \quad(\text { ecuación 3) }
$$

donde GP es el valor inicial después del acondicionamiento del transformador (950) y 2FAL es el valor de 2-furaldehído obtenido tanto por el método dirécto como el aproximado, en ppm.

Periodo de envejecimiento del transformador de potencia

El modelo matemático para la vida útil residual se basó en el periodo de envejecimiento (P.E) propuesto por [19]:

$$
P . E=\frac{\frac{1}{G P t}-\frac{1}{G P_{0}}}{A * 81760} * e^{b}[a \tilde{n} o s] \text { (ecuación 4) }
$$

donde $G P_{t}$ es el grado de polimerización crítico con valor de 200, $G P_{0}$ es el grado de polimerización estimado, $b=\frac{E_{a}}{R T}$ : donde $E_{a}$ es la energía de activación del proceso oxidativo del aceite dieléctrico regenerado $(111 \mathrm{~kJ} / \mathrm{mol}), R$ es la constante de gases ideales, $T$ es la temperatura en $\mathrm{K}$ medida en el aceite $A$ una constante de unidad de tiempo (horas) en función del contenido de oxígeno disuelto en el aceite (ppm) y de humedad en equilibrio (fracción decimal) del papel tipo kraft, calculado por las ecuaciones del cuadro 1.

Cuadro 1. Valores ecuaciones para el valor de $A$.

\begin{tabular}{|c|c|}
\hline Nivel de Oxígeno & A según el contenido de humedad "w" \\
\hline Bajo $(<7000 \mathrm{ppm})$ & $A=\left(1.78 \times 10^{12}\right) w^{2}+\left(1.10 \times 10^{10}\right) w+5.28 \times 10^{7}$ \\
\hline $\begin{array}{c}\text { Medio } \\
(7000-16500 \mathrm{ppm})\end{array}$ & $A=\left(2.07 \times 10^{12}\right) w^{2}+\left(5.61 \times 10^{10}\right) w+2.31 \times 10^{8}$ \\
\hline Alto $(>16500 \mathrm{ppm})$ & $A=\left(2.29 \times 10^{12}\right) w^{2}+\left(9.78 \times 10^{10}\right) w+3.86 \times 10^{8}$ \\
\hline
\end{tabular}

Fuente: Ecuaciones propuestas basadas en [13]. 
Vida útil residual

Una vez obtenido el periodo de envejecimiento del papel dieléctrico, la vida util residual $(N)$ se determinó como:

$$
N=35-P . E[a \tilde{n} o s] \text { (ecuación 5) }
$$

El número 35 se tomó como referencia de la vida útil establecida por los fabricantes de transformadores de potencia bajo condiciones nominales operativas.

\section{Resultados}

\section{Ensayos mecánicos de tensión}

A nivel general, las diferencias físicas entre las dos muestras de papel dieléctrico eran notables incluso antes de ser ensayadas, lo cual muestra grado de deterioro físico del papel dieléctrico usado por su color mucho más oscuro y translucido. Aun cuando ambas pruebas se efectuaron bajo las mismas condiciones, la diferencia en la forma de ruptura fue notable. La probeta de papel nueva siguió una dirección de fractura perpendicular a la fuerza aplicada, cualidad característica y esperada para tales condiciones. Debido a la naturaleza interfibral existente, los esfuerzos mecánicos del papel dieléctrico a su inicio de su vida recaen directamente en sus de Hemicelulosa y los distribuyen mejor a lo largo de la cadena molecular principal. En cambio, la muestra deteriorada no mostró un comportamiento similar, ya que su dirección de ruptura continuó la forma de arrollamiento de los conductores. A raíz de su fatiga mecánica, estas zonas demuestran una alta afinidad de fragilización donde a nivel molecular, las incisiones en la cadena principal son mayores y su grado de polimerización es menor, reflejado en su disminución de esfuerzos medidos (ver cuadro 2 y figura 1).

Cuadro 2. Resultados obtenidos del ensayo mecánico de tensión.

\begin{tabular}{|c|c|c|c|}
\hline Probeta & Esfuerzo último $(\mathrm{MPa})$ & \% de Reducción & GP estimado \\
\hline Nuevo & 56.94 & 28.83 & 348.70 \\
\hline Usado & 20.41 & 74.49 & 88.43 \\
\hline
\end{tabular}

La probeta de papel nueva presentó mejores respuestas mecánicas respecto a la del papel usado, pero no las esperadas para su condición. Si bien su grado de polimerización estimado aún se encuentra en un rango operativo [8] y su porcentaje de reducción aún no es objeto de análisis al no haber alcanzado un valor de disminución del 50\%, condición de rechazo por la IEC, es probable que sea el inicio de un deterioro crítico incipiente. La razón de tal situación tiene explicación por su condición de almacenamiento. Un mal control ambiental provoca la aceleración del proceso degradativo por su alto grado higroscópico. La hidrólisis provoca rupturas homolíticas en cadenas más pequeñas y la disminución del grado de polimerización. Esto sumado con los altos niveles de humedad locales, aportan a la reducción de la respuesta mecánica junto con el deterioro operativo normal. Por otro lado, el papel usado mostró una reducción del 74.49\% de su esfuerzo de tensión y un grado de polimerización crítico de 88.43. Estas características son suficientes para demostrar la deficiente condición de dicho papel. Si bien las pruebas mecánicas aplicadas al papel brindan información valiosa de la condición del transformador de potencia, su carácter invasivo lo hace impráctico en este tipo de tecnologías dieléctricas. 


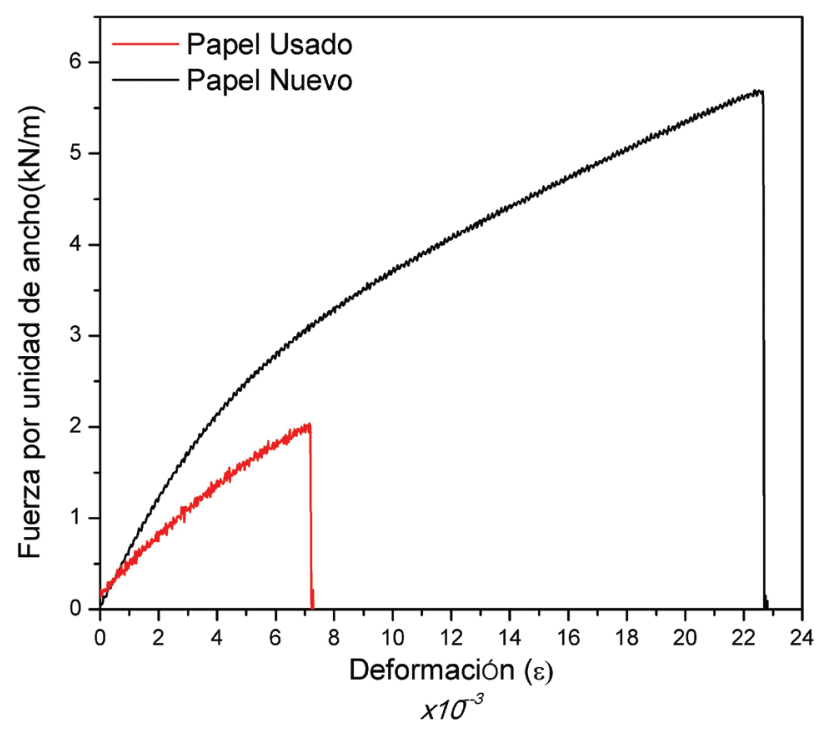

Figura 1. Curvas obtenidas de ensayos de tracción para ambas muestras de papel dieléctrico basadas en datos de investigación. Fuente: Resultados obtenidos de investigación.

Contenido de gases disueltos

Los transformadores de potencia mostraron un contenido de gases totales promedio de 83139 ppm, donde el $18.06 \%$ y $0.02 \%$ representaron el Oxígeno e Hidrógeno disuelto respectivamente. Los bajos niveles de Hidrógeno señalan una excelente hermeticidad de la parte activa con la intemperie, cualidad reflejada igualmente en las curvas de equilibrio de humedad (ver figura 2), estimadas de [17].

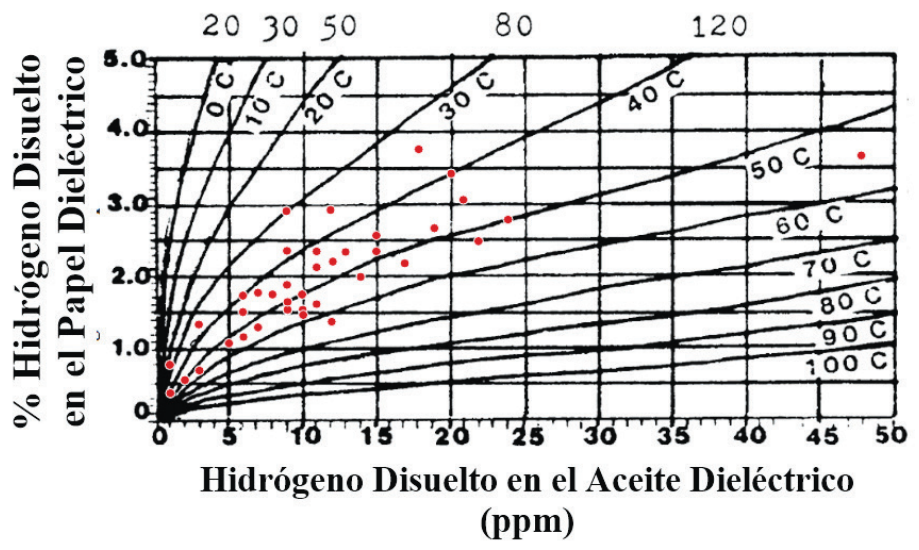

Figura 2. Puntos experimentales del contenido en equilibrio de agua en el papel dieléctrico estimados por la aplicación web WebPlotDigitizer y las curvas de [17].

Además de su baja naturaleza de solubilidad en el aceite (ver cuadro 3), el origen del contenido de Hidrógeno se deriva del mecanismo de degradación molecular en el aceite, por la producción de 2 moléculas de agua (humedad) en cada incisión homolítica. Esta consideración se correlaciona con el contenido de Oxígeno, debido a que este supera su límite de solubilidad solo en 2.06\% (309.31 ppm). Los niveles de exceso no son los suficientes para considerar una aceleración del proceso oxidativo normal en el aceite, el cual se incrementa exponencialmente 
a partir de un excedente en 2000 ppm. De modo que, los resultados comparados con el cuadro 3, determinan un deterioro normal en el aceite y baja producción de humedad interna, congruente igualmente a una buena hermeticidad.

Cuadro 3. Gases producidos por falla en el transformador

\begin{tabular}{|c|c|c|c|c|}
\hline \multirow{2}{*}{ Gas } & \multirow{2}{*}{ Solubilidad $^{2}$} & \multicolumn{3}{|c|}{ Rango de Supervisión (ppm) } \\
\hline & & Normal & Precaución & Alerta \\
\hline $\mathrm{H}_{2}{ }^{1}$ & $7.0 \%$ & $<100$ & $100-700$ & $>700$ \\
\hline $\mathrm{N}_{2}$ & $8.6 \%$ & & & \\
\hline $\mathrm{CO}^{1}$ & $9.0 \%$ & $<350$ & $350-570$ & $>570$ \\
\hline $\mathrm{O}_{2}$ & $16.0 \%$ & & & \\
\hline $\mathrm{CH}_{4}{ }^{1}$ & $30.0 \%$ & $<120$ & $120-400$ & $>400$ \\
\hline $\mathrm{CO}_{2}$ & $120.0 \%$ & & & \\
\hline $\mathrm{C}_{2} \mathrm{H}_{6}{ }^{1}$ & $280.0 \%$ & $<65$ & $65-100$ & $>100$ \\
\hline $\mathrm{C}_{2} \mathrm{H}_{4}{ }^{1}$ & $280.0 \%$ & $<50$ & $50-100$ & $>100$ \\
\hline $\mathrm{C}_{2} \mathrm{H}_{2}{ }^{1}$ & $400.0 \%$ & $<2$ & $2-5$ & $>5$ \\
\hline \multicolumn{5}{|c|}{${ }^{1}$ Gases Combustibles } \\
\hline \multicolumn{5}{|c|}{${ }^{2}$ Solubilidad a $101.33 \mathrm{kPa}$ y $25^{\circ} \mathrm{C}$} \\
\hline
\end{tabular}

Fuente: Datos obtenidos basados en [4].

El contenido de gases combustibles disueltos en el aceite trabaja es el parámetro de evaluación más utilizado por la atención especial de su evolución durante el periodo operativo del transformador. El cuadro 4 resume el $75 \%$ de las mediciones de gases combustibles disueltos del grupo de transformadores analizados.

Cuadro 4. Valores medidos del contenido gases combustibles.

\begin{tabular}{|c|c|c|}
\hline Gas & $75 \%(\mathrm{ppm})$ & Condición \\
\hline Hidrógeno & 16 & Normal \\
\hline Monóxido de Carbono & 506.5 & Precaución \\
\hline Metano & 15.5 & Normal \\
\hline Etano & 2 & Normal \\
\hline Etileno & 11.5 & Normal \\
\hline Acetileno & 0 & Normal \\
\hline
\end{tabular}

Fuente: Resultados obtenidos de investigación.

Es notable como, casi todos se encuentran en una situación de deterioro normal excepto el Monóxido de Carbono. La variación en el gas combustible sugiere problemas incipientes de descargas parciales, sobrecarga u operación en caliente del transformador, condiciones que favorecen la descomposición de celulosa (papel dieléctrico) y consecuentemente el incremento en los contenidos de Monóxido como producto de reacción. Lo anterior coloca en evidencia el efecto de la carga de trabajo y deterioro operativo que sufre el sistema dieléctrico en los transformadores, reflejado igualmente por la distribución de la figura 3. Los altos contenidos de gases preponderan en periodos extremos (entre 10-15 años y 30-45 años), lo cual se relaciona 
con la forma de probabilidad de fallo característica en los transformadores (forma de tina de baño). Asimismo, el incremento cinético y agilización de las reacciones de degradación fruto del aumento de temperatura, es evidente en la tendencia de formación de gases a mediciones superiores a los $40^{\circ} \mathrm{C}$. No obstante, la buena condición inclusive en equipos con periodos de operación superiores a los de vida útil estándar definido por los fabricantes (35 años), reafirman el impacto que conlleva un correcto mantenimiento durante su operación. Los bajos niveles de 2-FAL medidos, 0.0448 ppm, determinan una excelente condición (según [18]) y un bajo deterioro polimérico de la celulosa, con valores de GP aproximados de 660.

\section{Grado de Polimerización}

Las estimaciones del grado de polimerización para las mediciones directas e indirectas del 2-FAL fueron superiores a 857.84 y 708.6 respectivamente. En la figura 4 se muestra el resumen de ambas aproximaciones, donde es apreciable la diferencia de variabilidad. En vista a lo anterior, es de esperar que el cálculo con las mediciones de Furaldehídos normadas sean las más precisas pero en términos prácticos, la aproximación resulta ser una opción viable si se consideran aspectos logísticos del mantenimiento. Al tomar en cuenta el carácter no invasivo de la toma de muestras de aceites en los transformadores, el no intervenir directamente las partes activas reduce su contacto con la intemperie y mantiene invariable sus condiciones operativas. De igual forma, los costos asociados para el calculo del 2-FAL de forma directa son mayores a los de gases disueltos, principalmente en los consumibles, equipos y operación adicional requerida. Usualmente en las valoraciones del aceite dieléctrico se realizan análisis químicos de gases disueltos por medio de cromatografía de gases, en cambio, los furaldehídos por su solubilidad en el aceite y baja volatilidad, son medidos por cromatografía líquida de alta precisión. El valor agregado de la medición indirecta no solo permite reducir y agilizar los costos asociados, sino también controlar en todo momento la evolución de su condición por medio de la estimación de vida útil restante de los transformadores de potencia. Por lo cual, las medias aritméticas de 945.44 y 901.5 del grado de polimerización para las mediciones directas e indirectas respectivamente aún se encuentran en rangos evaluativos del envejecimiento en el papel dieléctrico (levemente envejecidos), útiles en aspectos ingenieriles para la toma de decisiones.

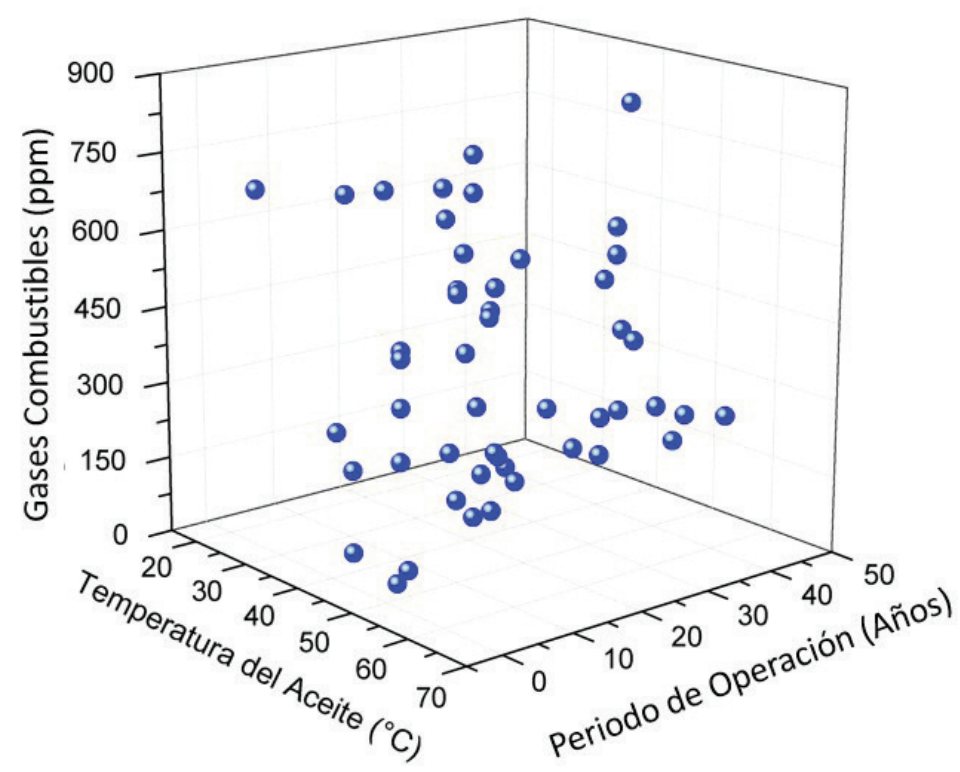

Figura 3. Dispersión de datos de gases combustibles totales para el conjunto de transformadores de potencia estudiados. 


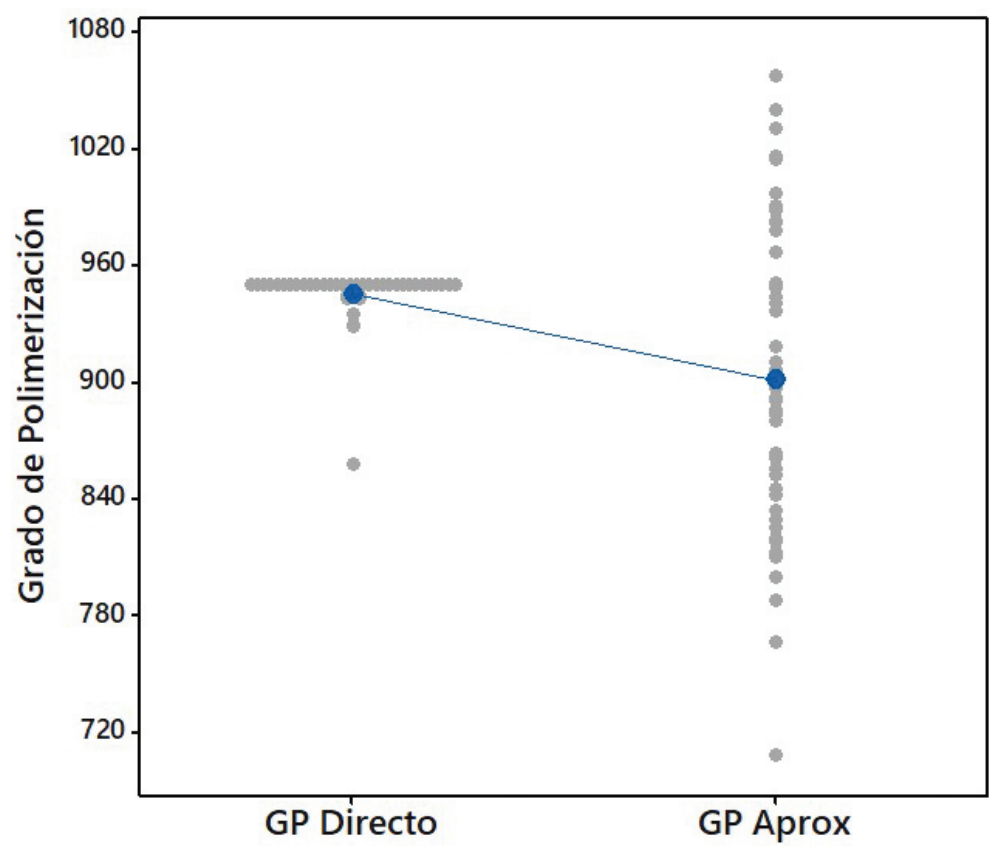

Figura 4. Gráfica de valores individuales para ambos métodos y sus respectivas medias aritméticas obtenidas de datos experimentales de la investigación.

\section{Estimación de vida útil residual}

En cuanto a los periodos residuales estimados con los dos métodos de aproximación del grado de polimerización, se comprobó, por medio de una prueba de t pareada, que hay probabilidad de que no existan diferencias representativas. Gracias el software estadístico MINITAB, la hipótesis alterna planteada, la cual considera diferencias entre los grupos de datos dentro de un intervalo de confiabilidad del 95\%, fue rechazada al presentar un valor de p superior al nivel de significancia (0.05). Este método, al demostrar la capacidad de comparar diferentes metodologías a partir de sus resultados, permitió evaluar el alcance de las estimaciones realizadas, aspecto importante al estar respaldado estadísticamente. Esto corrobora la practicidad del uso de indicadores o métodos indirectos en la estimación de vida útil residual, además del alcance del modelo matemático por sus ajustes exponenciales según sus contenidos de humedad y Oxígeno disuelto en las partes activas. Los valores de vida útil residual obtenidos mostraron periodos inferiores a 27 años para el $75 \%$ del conjunto de transformadores analizados, todos razonables para sus altos valores de grado de polimerización (evaluados a partir del 2-FAL).

Sin embargo, algunos transformadores presentaron periodos restantes entre los 5-10 años, esto a los contenidos de Oxígeno, humedad y temperatura medida del aceite dieléctrico. Debido a la incorporación de valores exponenciales que varían según estos elementos, como la variable A, ajustan y modelan las estimaciones matemáticas a condiciones reales cinéticas. La figura 5 muestra la distribución de las variables ajustadas para los transformadores analizados, donde la mayoría se encuentran en rangos de bajos contenidos de Oxígeno. Si bien el grado de polimerización ronda en valores entre 940-960, la presencia o combinación de estos 3 factores en el transformador incrementan la tasa degradativa y reducen su vida útil considerablemente. De modo que, su impacto dentro de un mantenimiento preventivo-correctivo aplicado a estos equipos, permiten no solo asegurar su operación confiable dentro de su ciclo de servicio, sino prolongar y maximizar su uso, bajo un monitoreo de su evolución. 


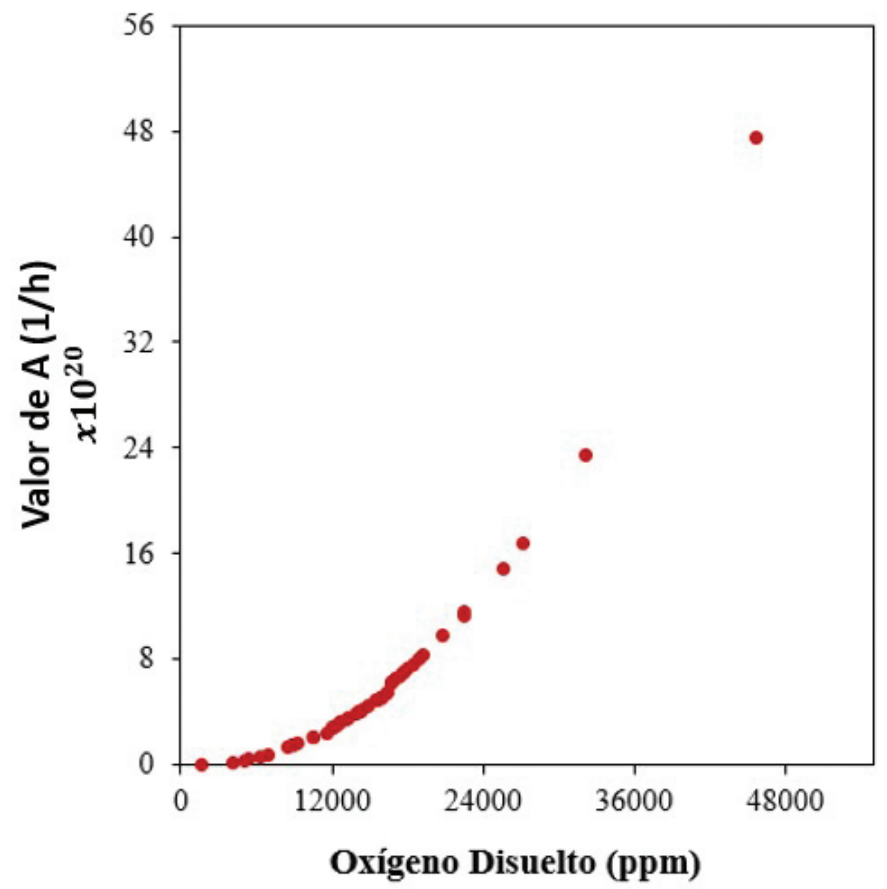

Figura 5. Valores de A en función del contenido de Oxígeno disuelto en el aceite y Humedad en equilibrio del papel dieléctrico de los transformadores analizados.

\section{Conclusiones}

El papel dieléctrico usado mostró un esfuerzo de tensión menor al nuevo, con un valor de 20.41 MPa. Lo anterior se atribuye al rompimiento homolítico de cadenas unitarias las cuales no actúan como una sola y disminuyen sus prestaciones mecánicas. En cuanto a la muestra de papel dieléctrico nuevo, aun cuando presentó una reducción en su esfuerzo de tensión de solo el $28.83 \%$ respecto a los valores estandarizados por la IEC, el grado de polimerización aproximado (348.70) comprueba el posible inicio de un deterioro crítico. Los bajos contenidos medidos de Hidrógeno disueltos en el aceite dieléctrico señalan la hermeticidad en los transformadores, donde su origen se explica por el mecanismo de deterioro del aceite dieléctrico en condiciones normales de envejecimiento. De igual forma el contenido de Oxígeno disuelto reafirmó dicha condición de deterioro la cual, a pesar de encontrarse en mayor proporción a su solubilidad (incremento de 309.31 ppm), su exceso no es suficiente para acelerar la degradación del aceite dieléctrico. La baja producción de gases combustibles generados acredita la buena condición en el sistema dieléctrico, junto con los insuficientes niveles de 2-furfural obtenidos (contenido medio de 0.0448 ppm) que puedan ser relacionados con una alta degradación de la celulosa inclusive a largos periodos operativos. Es así como el grado de polimerización estimado en ambos métodos fue superior a 708.6, condición que se relaciona con un leve deterioro en el dieléctrico sólido. Si bien estadísticamente los datos obtenidos por las diferentes metodologías de estimación del grado de polimerización mostraron cierto nivel de variabilidad, para efectos prácticos en estudios de la condición de los transformadores son suficientes, ya que ambos casos se encuentran dentro del mismo rango evaluativo reportado en investigaciones similares (útil para la toma de decisiones futuras). Finalmente, el 75\% de los transformadores presentaron una vida útil residual máxima de 27 años según el método matemático de envejecimiento utilizado. Dicho modelo demostró su capacidad de cálculo para cualquiera de los dos procesos de aproximación del grado de polimerización sin alguna diferenciación estadística relevante. 


\section{Referencias}

[1] R. Polansky et al, "Comparison of the mineral oil lifetime estimates obtained by differential scanning calorimetry, infrared spectroscopy, and dielectric dissipation factor measurements," Thermochimica Acta, vol. 647, pp. 86-93, 2017.

[2] A. Moreno Villa, "Estandarización E Implementación De La Norma ASTM D-4243 Para La Determinación Del Grado De Polimerización Del Papel Eléctrico (Nuevo Y Usado) Como Aislante En Transformadores De Potencia Por El Método Del Promedio Viscosimétrico;", Universidad Tecnológica de Pereira.

[3] Margalló Gasco, "Diagnóstico Del Consumo De Vida De Un Transformador a Través Del Análisis De Compuestos Furánicos.", 2012.

[4] H. M et al, Guía Para El Mantenimiento Del Transformador. (3era ed ed.) Estados Unidos: Transformer Maintenance Institute, 2005.

[5] R. Córdoba Salazar, "Desarrollo De Un Programa De Mantenimiento Basado En Confiabilidad Para Transformadores De Potencia.", Universidad de Costa Rica, 2006.

[6] M. Emsley et al, "Degradation of cellulosic insulation in power transformers. Part 4: Effects of ageing on the tensile strength of paper," IEE Proceedings - Science, Measurement and Technology, vol. 147, (6), pp. 285290, 2000.

[7] D. J. T. Hill et al, "A study of degradation of cellulosic insulation materials in a power transformer. Part 2: tensile strength of cellulose insulation paper," Polymer Degradation and Stability, vol. 49, (3), pp. 429-435, 1995.

[8] C. Homagk, K. Mossner and T. Leibfried, "Investigation on degradation of power transformer solid insulation material," in Oct 2008.

[9] G. Jiménez-Araya and G. A. Gómez-Ramírez, "Comportamiento de los aislamientos sólidos de transformadores de potencia en condiciones ambientales no controladas," Revista Tecnología En Marcha, vol. 29, (3), 2016.

[10] M. Emsley, "Review of chemical indicators of degradation of cellulosic electrical paper insulation in oil-filled transformers," IEEE Proceedings - Science, Measurement and Technology, vol. 141, (5), pp. 324, 1994.

[11] Gholami, M. Mirzaie And H. R. Tayebi, "Insulation condition assessment of power transformers using accelerated ageing tests," Turkish Journal of Electrical Engineering and Computer Sciences, vol. 17, (1), pp. 39-54, 2009.

[12] D. J. T. Hill et al, "A study of degradation of cellulosic insulation materials in a power transformer. Part 2: tensile strength of cellulose insulation paper," Polymer Degradation and Stability, vol. 49, (3), pp. 429-435, 1995.

[13] D. Martin et al, "An Updated Model to Determine the Life Remaining of Transformer Insulation," IEEE Transactions on Power Delivery, vol. 30, (1), pp. 395-402, 2015.

[14] Johana Tatiana Sarria-Arias, Natalia Andrea Guerrero-Bello and Edwin Rivas-Trujillo, "Estado del arte del análisis de gases disueltos en transformadores de potencia," Revista Facultad De Ingeniería, vol. 23, (36), pp. 105-122, 2014.

[15] W. Flores et al, "Vida de transformadores de potencia sumergidos en aceite: Situación actual. Parte II. Correlación entre resultados de ensayos físico-químicos," IEEE Latin American Transactions, vol. 5, (8), 2007.

[16] Hillary, W. D. A. G et al, "A tool for estimating remaining life time of a power transformer," in May 2017.

[17] T. V. Oommen, "Moisture equilibrium in paper-oil insulation systems," in Oct 1983

[18] F. Ortiz et al, "Estimating the age of power transformers using the concentration of furans in dielectric oil," Renewable Energy and Power Quality Journal, pp. 1011-1015, May 2016.

[19] R. A. Prasojo et al, "Transformer Paper Expected Life Estimation Using ANFIS Based on Oil Characteristics and Dissolved Gases (Case Study: Indonesian Transformers)," Energies, vol. 10, (8), pp. 1135, 2017. 